\title{
Centre of mass determination based on an optical weighing machine using fiber Bragg gratings
}

\author{
Rui Oliveira ${ }^{\mathrm{a}}$, Paulo Roriz ${ }^{\mathrm{a}, \mathrm{b}}$, Manuel B. Marques ${ }^{\mathrm{a}, \mathrm{c}}$, Orlando Frazão ${ }^{1 \mathrm{a}, \mathrm{c}}$ \\ ${ }^{a}$ INESC TEC, Rua do Campo Alegre 687, 4150-179 Porto, Portugal; ${ }^{b}$ Institute University of Maia \\ (ISMAI), Av. Carlos Oliveira Campos, Castelo da Maia, 4475-690 Maia, Portugal; Dept. of Physics \\ and Astronomy, Faculty of Sciences, University of Porto, Rua do Campo Alegre 687, 4169-007, \\ Porto, Portugal.
}

\begin{abstract}
The purpose of the present work was to construct a weighing machine based on fiber Bragg gratings (FBGs) for the location of the 2D coordinates of the center of gravity (COG) of objects with complex geometry and density distribution. The apparatus consisted of a rigid equilateral triangular platform mounted on three supports at its vertices, two of them having cantilevers instrumented with FBGs. As an example, two femur bone models, one with and one without a hip stem prosthesis, are used to discuss the changing of the COM caused by the implementation of the prosthesis.
\end{abstract}

Keywords: Optical fiber Sensors, fiber Bragg gratings, Cantilever, Centre of mass, hip prosthesis

\section{INTRODUCTION}

The center of gravity (COG) is a point in space where the weight of a body is concentrated. Finding the coordinates of the COG has many applications, namely in biomechanics. However, the location of the COG of the whole body, or a body segment (e.g., arm, forearm, and others) or even a prosthesis, could be a demanding task due to their 3D complex geometry and the heterogeneity of the materials that compose them. Moreover, in the case of the body segments, which are connected through the joints in a continuous fashion, accurate measurements such as of the mass or weight of a single segment could be a cumbersome task because the segment ends or boundaries are not clearly distinguishable. In fact, depending on the assumptions used to define the segment landmarks the COG location could change ${ }^{1}$.

The cantilever-based designs are suitable for low frequency measurements with high sensitivity and low cross-axis sensitivity. The cantilever based fiber Bragg grating (FBG) structure basically comprises of a vibrating cantilever with an FBG bonded on the surface or attached to it. The bending strain of the cantilever is transferred to the FBG which results in a wavelength shift proportional to strain. Usually this type of structure is used as accelerometer due to its wide application to seismic and civil structural measurements ${ }^{2,3}$. However, present work aims to present a solution for the COM determination based on optical weighing machine using FBG sensors.

\section{EXPERIMENTAL SETUP AND RESULTS}

The experimental setup consisted of a rigid equilateral triangular platform mounted on three supports at its vertices, two of them with a cantilever instrumented with a FBG, the other supporting the

\footnotetext{
${ }^{1}$ ofrazao@inescporto.pt
}

24th International Conference on Optical Fibre Sensors, edited by Hypolito José Kalinowski, José Luís Fabris, Wojtek J. Bock, Proc. of SPIE Vol. 9634, 96347H · (c) 2015 SPIE CCC code: $0277-786 X / 15 / \$ 18 \cdot$ doi: $10.1117 / 12.2195151$ 
reaction platform directly (Figure 1). The FBGs were used to measure the amount of bending strain at the cantilever caused by an object's weight placed on the platform. For calibration purposes the shift on the Bragg's wavelength $\left(\lambda_{B}\right)$ was plotted against weight variation using circular calibrated weights carefully put over the reaction platform barycenter. Therefore, the weight would be evenly distributed over the 3 vertices of the triangle making the calibration process easier. The characterization for both FBGs is shown in Figure 2.

The FBGs were interrogated using a FiberSensing BraggMeter (www.fibersensing.com). Additionally, a video camera was used for capturing an image of the object over the platform and drawing the COG point automatically. All the signal reading along with the image from the camera have been processed on LabView software.

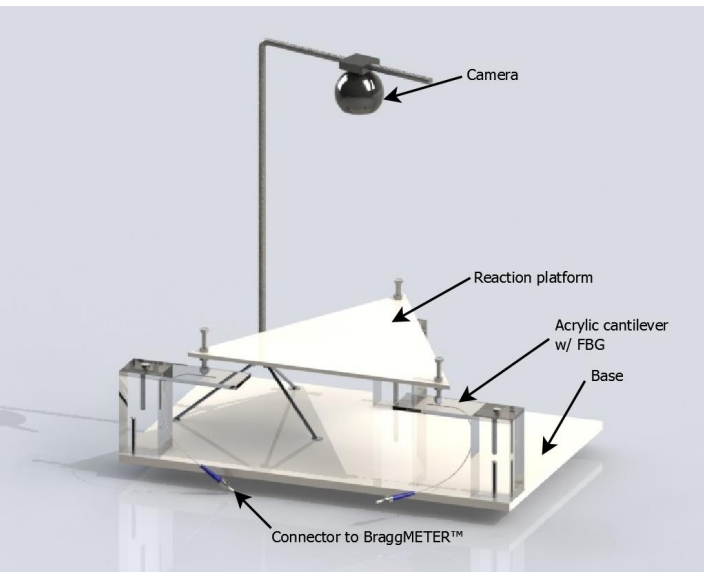

Figure 1. Scheme of the used setup featuring the camera, the reaction platform and the cantilevers.

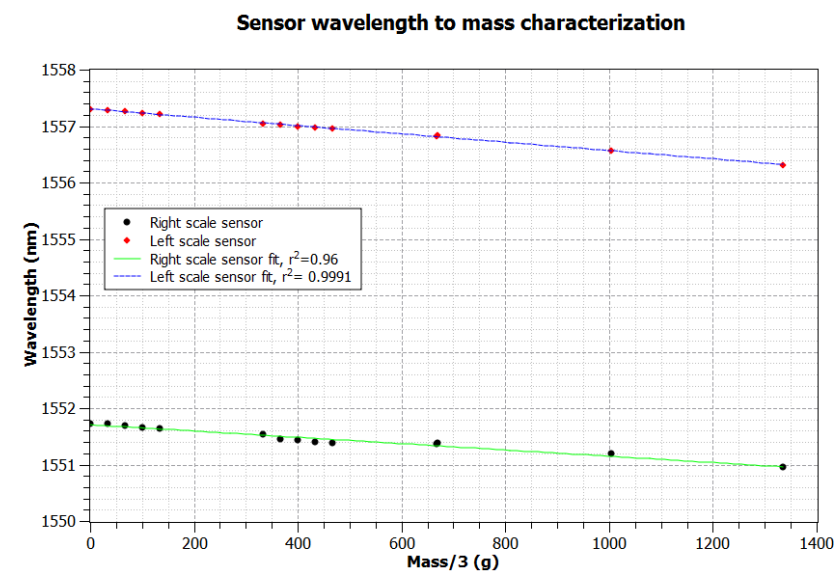

Figure 2. Characterization of the FBGs over the applied mass. Mass on the $\mathrm{x}$ axis is $1 / 3$ of the mass of the calibrated weight used in the experiment.

The maximum vertical displacement at a distance $L$ was a load with an applied weight, $P$, and is given by $\delta=1 / 3(P L / E I)$, where $E$ is the Young's Modulus and $I$ is the moment of inertia, given by $I=w h^{3} / 12$. Here it is considered a prismatic cantilever beam with a rectangular section (depth $h$ and width $w$ ). The dependence of the wavelength variation with the applied weight over each scale presents linear response with negative slopes due to the compression of the FBG when is used in the cantilever and the sensitivities are: $-7.36 \times 10^{-4} \mathrm{~nm} / \mathrm{grams}$ and $-5.59 \times 10^{-4} \mathrm{~nm} / \mathrm{grams}$ on the left and right FBGs respectively. The resolution of the resultant weighting machines is 58.12 grams. The coordinates of the COG are determined using equations derived from the equilibrium of forces and moments of forces on the balances, according to the following equation:

$$
c_{1}=\frac{\frac{c}{2}\left(-f_{r}^{A}+p-f_{r}^{B}\right)+c \times f_{r}^{B}}{p}, b_{1}=\frac{\frac{b}{2} \times f_{r}^{B}+b \times f_{r}^{A}}{p} ;
$$

Where $f_{r}^{x}, x=A, B$ is the force applied on each vertex and are equal to $f_{r}^{x}=m_{x} g, x=A, B, a$ is the side of the used equilateral reaction platform, and $c_{1}$ and $b_{1}$ are a first set of COG coordinates, natural to the reaction platform itself, that are after converted to match a point on the plot area of the image. The two calibration equation: $\lambda_{F B G 1}(\mathrm{~nm})=5.6 \times 10^{-4} m_{A}(\mathrm{~g})+1551.706$ and $\lambda_{F B G 2}(\mathrm{~nm})=7.3 \times 10^{-4}$ $m_{B}(\mathrm{~g})+1557.303$. 
Knowing the mass of the object itself, on LabView, three points calibrate the vertex of the reaction platform. A red point is then shown over the image of the reaction platform where COG was calculated. Figures $3 \mathrm{a}$ ) and $3 \mathrm{~b}$ ) show this process. On this particular work, it is used our setup to compare the COG of a regular human femur against a femur with a synthetic prosthesis on the joint with the hip. The first noticeable difference is the mass of the objects themselves: while the femur model weights $569 \mathrm{~g}$, its prosthetic equivalent weights $741 \mathrm{~g}$. Figures $3 \mathrm{a}$ ) and $3 \mathrm{~b}$ ) evidence the clear difference of the center of mass. Using the reaction platform as scale ( $0.30 \mathrm{~m}$ vertex to vertex), the difference between the centers of mass can be calculated around $47 \mathrm{~mm}$.

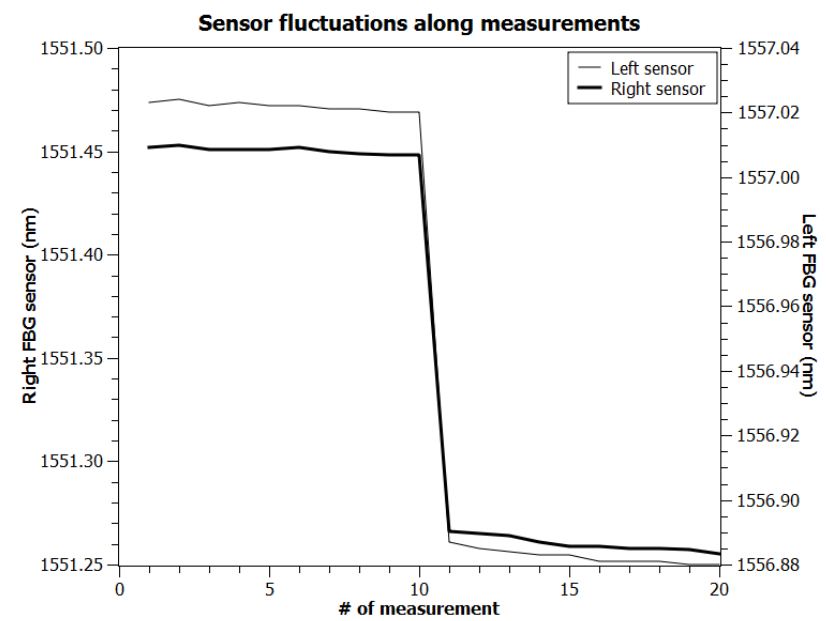

Figure 3. Sensor behavior along time featuring two different applied weights. Measurements were made with one second intervals.
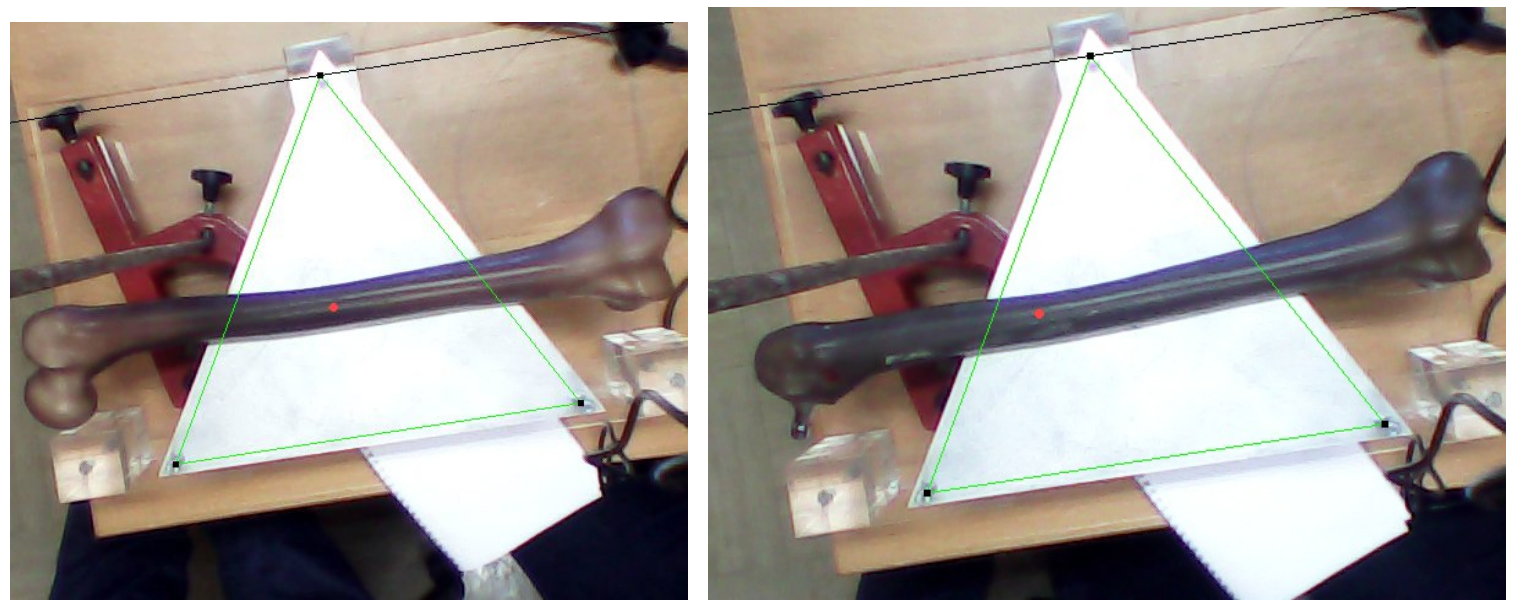

Figure 4. a) Bone without prosthesis and b) Bone with prosthesis.

\section{CONCLUSIONS}

Although the resolution on the weighting machine was beyond the desirable one, noticeable change on the COG of the prosthesis is clear. Future work might imply human sized reaction platform setups for full body measurements so that the effect of prosthesis on the center of mass of human beings can be studded and its implications discussed. It could be hypothesized that this imbalance of 
mass and COG distribution could change the biomechanical behavior of the segment and of the whole body, such as the distribution of body weight through the feet, the pattern of the ground reaction force and of the center of pressure, and the trajectory of the COG during locomotion. On the setup itself, improvements to cantilevers quality arises, so that sensor fluctuations can be reduced and therefore resolution can be increased as plastic relaxation phenomenon are the probable issues to blame on the fluctuations of the sensor, as they have a clear negative slope on each measurement.

\section{ACKNOWLEDGMENTS}

Project "NORTE-07-0124-FEDER-000058" is financed by the North Portugal Regional Operational Programme (ON.2 - O Novo Norte), under the National Strategic Reference Framework (NSRF), through the European Regional Development Fund (ERDF), and by national funds, through the Portuguese funding agency, Fundação para a Ciência e a Tecnologia (FCT).

\section{REFERENCES}

[1] HINRICHS, R. N. 1990. Adjustments to the segment center of mass proportions of Clauser et al. (1969). Journal of Biomechanics, 23, 949-951.

[2] M. D. Todd, G. A. Johnson, B. A. Althouse, and S. T. Vohra, "Flexural beam-based fiber Bragg grating accelerometers", IEEE Photonics Technology Letters, Vol. 10, No. 11(1998).

[3] T. A. Berkoff and A. D. Kersey, "Experimental demonstration of a fiber Bragg grating accelerometers, IEEE Photonics Technology Letters, Vol. 8, No. 12 (1996). 